\title{
Efficient right ventricular shape modeling using a dual active shape model
}

\author{
Hossam El-Rewaidy ${ }^{2}$, El-Sayed H Ibrahim ${ }^{1 *}$, Ahmed S Fahmy ${ }^{2}$ \\ From 19th Annual SCMR Scientific Sessions \\ Los Angeles, CA, USA. 27-30 January 2016
}

\section{Background}

Active shape models (ASM) showed to have potential for segmenting the right ventricle (RV) in CMR images. Nevertheless, the large variability and complexity of the RV shape do not allow for concisely capturing all possible shape variations among different patients and anatomical cross-sections. Noticeably, the latter increases the number of iterations required to converge to a proper solution and reduces the segmentation accuracy.

\section{Methods}

In this work, a new ASM framework has been developed for efficiently modeling the RV shape in short-axis CMR images. In this framework, the RV contour is split into two simpler segments, septal and free wall, whose shape variations are independently modeled using two separate (dual) ASM models Figure 1(a). The contour splitting is done at the location of the RV insertion points into the septal wall. Further, instead of using the conventional
Procrustes method, the RV contours are aligned using the Bookstein coordinate transformation, which uses the $\mathrm{RV}$ insertion points as landmarks to nonlinearly align the RV contours (Figure 1(b)). The proposed framework allows for building one ASM model from all short-axis slices. The developed technique has been tested on a dataset from 10 patients imaged with cine CMR.

\section{Results}

Table 1 shows the mean \pm SD errors between the contours produced by the proposed and conventional ASM models with respect to manually delineated contours at the three different cross-sectional slices. As can be seen in Table 1, the performance of the proposed ASM framework is better than that of the conventional ASM model. This is evident by the lower value of the Mean Absolute Distance (MAD) and Hausdorf measures and higher value of the Dice index. Figure 1(c) shows the evolution of the ASM models from the initial contour

\begin{tabular}{|c|c|c|c|c|c|c|c|c|c|}
\hline & \multicolumn{3}{|c|}{ MAD } & \multicolumn{3}{|c|}{ Hausdorff } & \multicolumn{3}{|c|}{ Dice Index } \\
\hline & Basal & Mid $^{*}$ & Apical $^{+}$ & Basal & Mid $^{*}$ & Apical $^{+}$ & Basal & Mid $^{\wedge}$ & Apical $^{+}$ \\
\hline $\begin{array}{c}\text { Proposed } \\
\text { ASM }\end{array}$ & $4.4 \pm 4.5$ & $4.7 \pm 4.2$ & $4.9 \pm 5.5$ & $7.8 \pm 7$ & $8.5 \pm 6.3$ & $9.5 \pm 8.9$ & $0.91 \pm 0.08$ & $0.90 \pm 0.09$ & $0.85 \pm 0.14$ \\
\hline $\begin{array}{c}\text { Conventional } \\
\text { ASM }\end{array}$ & $5.0 \pm 2.6$ & $5.9 \pm 3.3$ & $7.6 \pm 5.2$ & $8.6 \pm 4.6$ & $9.4 \pm 4.6$ & $12.0 \pm 7.6$ & $0.89 \pm 0.07$ & $0.87 \pm 0.07$ & $0.8 \pm 0.13$ \\
\hline $\begin{array}{l}{ }^{+} \text {p-value } \\
\text { re } 1 \text { Mean } \pm s \\
\text { cavity, and ap } \\
\text { nentation. }\end{array}$ & $\begin{array}{l}005 \text {; } \\
\text { the } r \\
\text { evels }\end{array}$ & he $n$ & d do & $\begin{array}{l}<0.0^{\circ} \\
\text { D), Has. } \\
\text { ISM an }\end{array}$ & rent & $\begin{array}{l}\text { Index } \\
\text { SM me }\end{array}$ & with $r$ & to & $\begin{array}{l}\text { urs at basal } \\
\text { th manual }\end{array}$ \\
\hline
\end{tabular}

'University of Michigan, Ann Arbor, Ml, USA

Full list of author information is available at the end of the article

(c) 2016 El-Rewaidy et al. This is an Open Access article distributed under the terms of the Creative Commons Attribution License (http://creativecommons.org/licenses/by/4.0), which permits unrestricted use, distribution, and reproduction in any medium, provided the original work is properly cited. The Creative Commons Public Domain Dedication waiver (http://creativecommons.org/ publicdomain/zero/1.0/) applies to the data made available in this article, unless otherwise stated. 


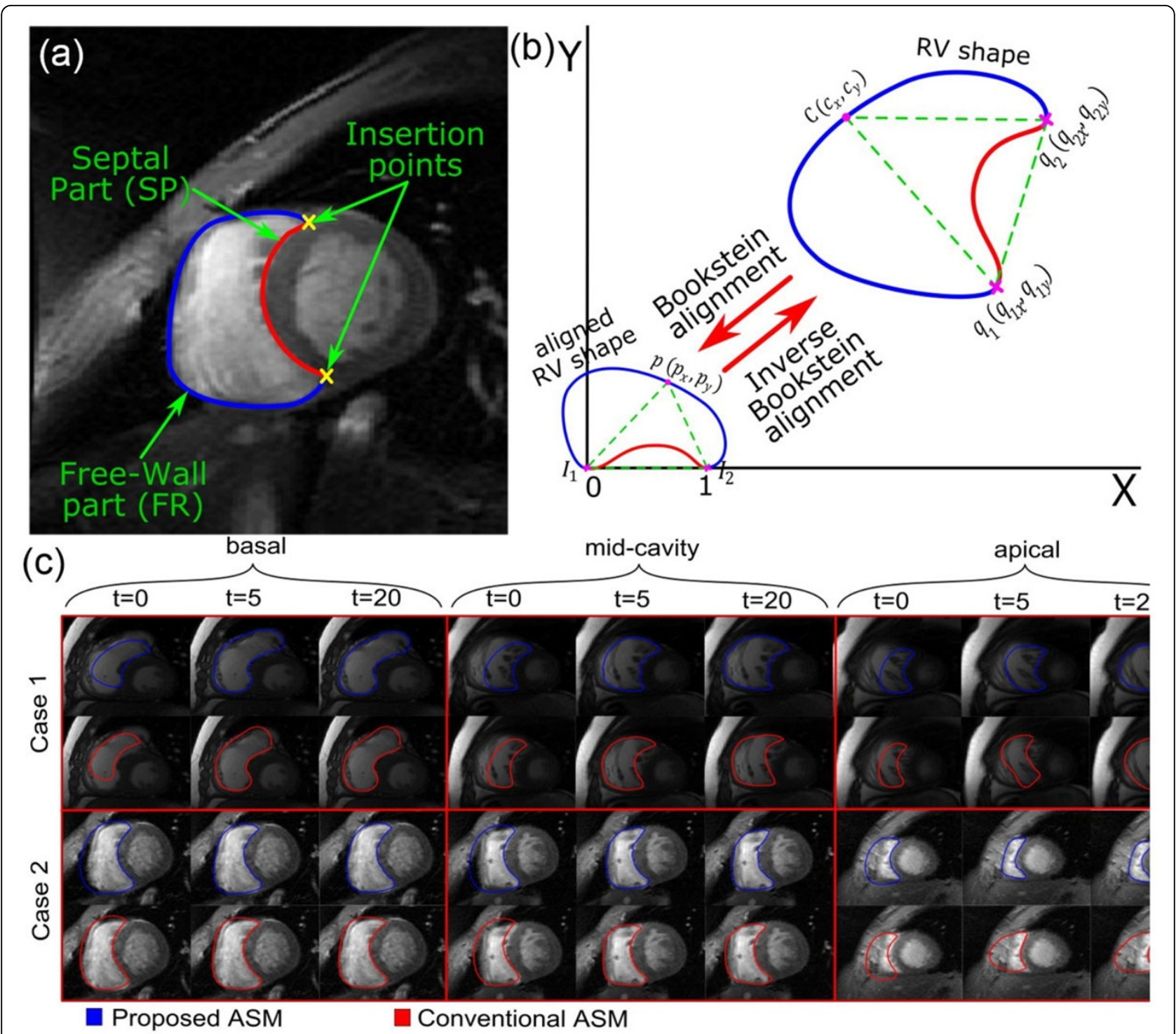

Figure 2 (a) The RV contour is divided into 2 segments (septal and free wall). (b) The transformation of the RV shape to the Bookstein Coordinates is performed in 2 steps: 1) registering the two insertion points to points 0 and 1 on the $x$-axis; and 2) normalizing each point on the original RV shape with respect to the distance between the insertion points. (c) The RV segmentation results in 2 cases using the proposed and conventional ASM models at the initial, 5th, and 20th interations. The figure shows 3 cross-sections at basal, mid-cavity and apical levels.

to the contours at iterations number 5 and 20 for two patients. It can be seen in the figure that the initial contour of the proposed ASM framework is much better than that of the conventional ASM model. The figure also shows that the proposed ASM framework converges after almost 5 iterations whereas the conventional ASM model needs 15-20 iterations to correctly delineate the RV contour. The average computation times for segmenting one slice using a personal computer were 0.09 $\mathrm{s}$ and $0.17 \mathrm{~s}$ for the conventional and proposed ASM models, respectively. Nevertheless, the parallelized nature of the problem renders this difference insignificant.

\section{Conclusions}

The developed double-ASM RV segmentation technique outperforms the conventional ASM framework and can efficiently model complex RV shape variation with more accuracy in less iteration steps. Although the proposed framework extracts only the RV endocardium, the epicardium can be segmented through dilating the endocardium contour.

Authors' details

${ }^{1}$ University of Michigan, Ann Arbor, MI, USA. ${ }^{2}$ Nile University, Cairo, Egypt. 

and take full advantage of:

- Convenient online submission

- Thorough peer review

- No space constraints or color figure charges

- Immediate publication on acceptance

- Inclusion in PubMed, CAS, Scopus and Google Scholar

- Research which is freely available for redistribution 